\title{
Quantum effect logic in cognition
}

\author{
Bart Jacobs \\ Institute for Computing and Information Sciences, Radboud University Nijmegen, P.O. Box 9010, 6500 GL Nijmegen, The Netherlands
}

\section{H I G H L I G H T S}

- Illustration of novel quantum effect logic in quantum cognition.

- Explanation of strengths of effect logic w.r.t. traditional projection-based logic.

- Demonstration of the (Python / EfProb) tool support of the effect logic.

- Re-description of standard quantum cognition examples with effect logic.

\section{A R T I C L E I N F O}

Article history:

Received 16 February 2017

Received in revised form 27 June 2017

Available online $\mathrm{xxxx}$

\begin{abstract}
A B S T R A C T
This paper illustrates applications of a new, modern version of quantum logic in quantum cognition. The new logic uses 'effects' as predicates, instead of the more restricted interpretation of predicates as projections - which is used so far in this area. Effect logic involves states and predicates, validity and conditioning, and also state and predicate transformation via channels. The main aim of this paper is to demonstrate the usefulness of this effect logic in quantum cognition, via many high-level reformulations of standard examples. The usefulness of the logic is greatly increased by its implementation in the programming language Python.
\end{abstract}

(C) 2017 Elsevier Inc. All rights reserved.

\section{Introduction}

Quantum physics is 'strange' and full of counter-intuitive phenomena. Accordingly, the mathematical foundations of quantum physics are 'strange', with observations having a side-effect, noncommutativity, and context-dependence. In the relatively new area of quantum cognition one tries to model aspects of human cognition using the mathematical formalism of quantum theory. Indeed, some judgements and choices that people make are also 'strange', see for instance the many illustrations in Kahneman (2011). The idea is that quantum logic provides more suitable 'laws of thought' than classical Boolean logic (Boole, 1854), leading to more realistic predictions about the type of errors that humans make.

We briefly mention some of the relevant connections.

- The 'conjunction and disjunction errors' in for instance the Linda example from Tversky and Kahneman (1983) can be described logically via the fact that quantum conjunction \&,

\footnotetext{
The research leading to these results has received funding from the European Research Council under the European Union's Seventh Framework Programme ( FP7/2007-2013)/ ERC grant agreement nr. 320571.

E-mail address: B.Jacobs@cs.ru.nl.

URL: http://www.cs.ru.nl/B.Jacobs.
}

and the associated disjunction, are not monotone in their first arguments.

- The relevance of the order of processing information is reflected by the non-commutativity of quantum conjunction \&.

- The order effect in inference and priming is mirrored by the non-commutativity of conditioning of quantum states.

- Different perspectives can be described via the contextdependence in quantum theory, given by a choice of basis in a vector (Hilbert) space.

- Answering a question creates a cognitive context in a way that can be compared to how measurement in quantum theory changes the state of an object under measurement.

This non-commutativity and context-dependence is the main reason for moving to quantum logic and probability in cognition research, see for instance the books by Busemeyer and Bruza (2012), Khrennikov (2010) for more information, references and discussion.

The main point of this paper is simple: if one decides to use quantum logic in cognition, then one might as well use the modern and expressive logic of effects, instead of the (restricted) logic of projections that is being used so far.

Historically, quantum logic started in the 1930s with Birkhoff and von Neumann (1936). They used a geometric interpretation 
of logical predicates as closed linear subspaces of Hilbert spaces. These subspaces have the algebraic structure of an orthomodular lattice (Kalmbach, 1983). Such subspaces can equivalently be described as projection operations on Hilbert spaces. In 1990s these projections have been generalised to 'effects', that is, to positive operation below the identity, which are used as interpretations of predicates, see Foulis and Bennett (1994) (and independently Chovanec and Kôpka (2007); Giuntini and Greuling (1994), see Dvurečenskij and Pulmannová (2000) for an overview). Such effects have the mathematical structure of an effect algebra/module and form the basis of a novel approach to quantum probability (Gudder, 2007). In order to emphasise the difference, projections are sometimes called 'sharp' predicates, and effects are the 'nonsharp' ones. Similarly, in classical (non-quantum) probabilistic logic one can call $\{0,1\}$-valued functions sharp predicates; they are contrasted with $[0,1]$-valued functions, forming the non-sharp, fuzzy predicates. A crucial difference is that non-sharp predicates are closed under sequential conjunction $\&$, under scalar multiplication, and under predicate transformation, as will be explained below.

In a further line of development it has been shown that effect module structure arises naturally from a certain elementary categorical structure, called an 'effectus', see Cho, Jacobs, Westerbaan, and Westerbaan (2015) and Jacobs (2015). These effectuses form a general framework, including Boolean logic, classical probabilistic logic, and quantum logic. The quantum case involves noncommutativity of conjunction \& and predicates that can have side-effects. The properly quantum effectuses form the abstract background for the current article. The main mathematical example is given by von Neumann algebras. Here we shall concentrate on more concrete examples, namely finite-dimensional Hilbert spaces-whose collections of operators form von Neumann algebras.

The main claim underlying this paper is that the quantum effect logic that started in the 1990s is more suitable for application in cognitive psychology, in comparison to the quantum subspace logic from the 1930s. This claim will be substantiated via several examples. These examples are not new, and in fact completely standard, taken from the well-known textbook (Busemeyer \& Bruza, 2012). This is a deliberate choice, so that (cognition) researchers who are already familiar with these examples can now recognise them in the new effect formalism. Hence this paper does not offer any new - mathematical or psychological - results or theories; instead, it offers a broader logical perspective, supported by an implementation for calculating probabilities.

Indeed, the abstract logic and probability theory of effectuses come with an implementation in the popular programming language Python, called EfProb, for 'Effectus Probability'. ${ }^{1}$ This implementation defines primitives for probability and logic which can be used to define states, predicates and channels, and to compute validities and conditioned states. Interestingly, EfProb has a uniform approach to discrete, continuous, and quantum probability. Here we shall only use the quantum fragment of the language: all the calculations in the examples below are obtained via EfProb . How this is done in Python is illustrated in Appendix, for two of the examples. The EfProb formalisation of all the examples is made available, so that interested readers can see the details, make variations, and adapt to their own setting.

We should emphasise that we use the word 'logic' as in Birkhoff and von Neumann (1936), referring to a mathematical interpretation, and not to a formal system of rules of inference. So far, such a formal, symbolic logic of effects does not exist. Nevertheless, we

\footnotetext{
1 EfProb is publicly available via the website efprob.cs.ru.nl, together with an extensive user manual. Kenta Cho and the current author are the main developers, see Cho and Jacobs (2017) for an overview.
}

consider predicates as effects on a Hilbert space, closed under a number of logical operations, including notably conjunction and predicate transformation. Similarly, states are density matrices on a Hilbert space, closed under certain operations, including conditioning and state transformation. In the current approach we can form composite predicates, via these logical operations, for expressing different statements, such as "feminist \& bank-teller" or "feminist | bank-teller" in the Linda example. Such complex predicates can be used to represent the situation at hand in logical form. Subsequently we can calculate the validity of such composite predicates.

In the remainder of this paper we describe in a step-by-step manner the main ingredients of the logic and probability theory of effectuses. Illustrations play an important role and are provided along the way. The reader is assumed to be familiar with the basics of the Hilbert space formalism of quantum theory, see e.g. Nielsen and Chuang (2000), Rieffel and Polak (2011) or Busemeyer and Bruza (2012) and Yearsley and Busemeyer (2016). The exposition starts with the basic notions of state and predicate, and with the validity of a predicate in a state, expressed as probability, in the unit interval $[0,1]$. This can be used immediately in the famous Linda example. After this illustration, some more background information about effects is given in Section 3, including a brief comparison to classical probabilistic logic using fuzzy predicates. Section 4 introduces conditioning (update, revision) of a state by a predicate, and illustrates this construct in the well-known polarisation example from physics. Subsequently, Section 5 describes channels, and forward state transformation and backward predicate transformation along a channel. These transformations are illustrated in an example involving a man and a woman with different perspectives on car brands. Finally, Section 6 illustrates how the relevance of the order of conditioning quantum states can be used to describe rulings in a court case depending on the order in which evidence is presented.

\section{States, predicates and validity}

This section briefly introduces states and predicates, and illustrates how they are used in the Linda example.

States and predicates form the basic ingredients of our framework. It is important to distinguish them clearly, since they play completely different roles and have different operations. For instance, predicates are closed under multiplication with a scalar from the unit interval $[0,1]$, but states are not. Intuitively, a state $\omega$ captures a certain state of affairs, and a predicate $p$ captures a property of that state. We shall write $\omega \vDash p$ for validity: the probability, expressed as a number in the unit interval $[0,1]$, that property $p$ holds in the state $\omega$. This validity $\omega \vDash p$ may also be read as the expected value of property $p$ in state $\omega$.

Let $\mathscr{H}$ be a finite-dimensional complex Hilbert space. A state $\omega$ of $\mathscr{H}$ is a positive operator on $\mathscr{H}$ with trace one. That is, $\omega$ is linear function $\omega: \mathscr{H} \rightarrow \mathscr{H}$ satisfying $\omega \geq 0$ and $\operatorname{tr}(\omega)=1$. A state is often called a density matrix. The canonical way to define a state is to start from a vector $|v\rangle \in \mathscr{H}$ with norm 1 , and consider the operator $|v\rangle\langle v|: \mathscr{H} \rightarrow \mathscr{H}$. It sends an arbitrary element $|w\rangle \in \mathscr{H}$ to the vector $\langle v \mid w\rangle \cdot|v\rangle$. An arbitrary state is a convex combination of such vector states $|v\rangle\langle v|$.

A predicate, also called an effect, is a positive operator $p$ on $\mathscr{H}$ below the identity: $0 \leq p \leq$ id. The identity id is given by the identity/unit matrix, and corresponds to the truth predicate, often written as 1. For each predicate $p$ there is an orthosupplement, written as $p^{\perp}$ or as $\sim p$, playing the role of negation. It is defined by $\sim p=$ id $-p$, and satisfies: $\sim \sim p=p$ and $p+\sim p=\mathbf{1}$. Section 3 gives more mathematical background information about predicates; at this stage we concentrate on how they are used. 
The most interesting logical operation on quantum predicates is sequential conjunction \&. It is defined via the square root operation on predicates, as:

$p \& q=\sqrt{p} q \sqrt{p}$.

We pronounce $\&$ as 'and-then', and read it as: after $p$ with its sideeffect, the predicate $q$ holds. As we shall see below in (4), \& is not commutative (and also not associative). This operation \& has been studied well before effectus theory, see e.g. Gudder and Greechie (2002). There is an associated 'or-then' operation |, defined as 'De Morgan' dual, namely:

$p \mid q=\sim(\sim p \& \sim q)$.

As we will see, for instance in Eq. (9) below, projections are not closed under \& and |, showing that these conjunction and disjunction operations cannot exist in the logic of Birkhoff and von Neumann (1936). Hence if we wish to use a logic with \& and I, we are forced to extend our interpretation of 'predicate' from projections to effects.

Mathematically, a state is also a predicate, but not the other way around. ${ }^{2}$ In the examples that we adapt from the literature, the predicate definitions look precisely the same as state definitions. But more generally, predicates can be defined in other ways too.

States $\omega$ and predicates $p$ of the same Hilbert space $\mathscr{H}$ can be combined in validity, defined as:

$\omega \vDash p=\operatorname{tr}(\omega p) \in[0,1]$.

This standard definition is also known as the Born rule.

With these definitions in place we can already describe the famous Linda example from Tversky and Kahneman (1983).

Example 1. We illustrate sequential conjunction \& and disjunction | in the Linda problem leading to 'conjunction fallacies' and 'disjunction fallacies'. We follow the description in Busemeyer and Bruza (2012). Consider the following quote and subsequent questions.

Linda is 31 years old, single, outspoken and very bright. She majored in philosophy. As a student, she was deeply concerned with issues of discrimination and social justice, and also participated in anti-nuclear demonstrations.

What is the likelihood of the following events? Linda is:

(i) active in the feminist movement;

(ii) a bank teller;

(iii) active in the feminist movement, and a bank teller;

(iv) a bank teller, or active in the feminist movement.

The 'conjunction fallacy' concerns the fact that when asked, many people say that option (iii) is more likely than option (ii), and the 'disjunction fallacy' occurs when option (iv) is judged to be less likely than option (i).

We illustrate how to realise these fallacies in a quantum setting, using the framework of states and predicates sketched above, in particular using the sequential conjunction $\&$ and $\mid$ in a compositional manner for the above points (iii) and (iv). We start from the following (vector) state $\omega$.

$$
\begin{aligned}
\omega=|v\rangle\langle v|=\left(\begin{array}{ll}
0.976 & -0.155 \\
-0.155 & 0.024
\end{array}\right) \\
\text { where }|v\rangle=\left(\begin{array}{c}
0.987 \\
-0.1564
\end{array}\right) \in \mathbb{C}^{2} .
\end{aligned}
$$

The predicates fem for 'feminist' and btr for 'bankteller' are also obtained from vectors.

\footnotetext{
2 The fact that states are predicates happens to hold for Hilbert state models, but fails for more general von Neumann algebras.
}
fem $=|u\rangle\langle u|=\left(\begin{array}{ll}1 & 0 \\ 0 & 0\end{array}\right)$
btr $=|w\rangle\langle w|=\left(\begin{array}{ll}0.095 & 0.293 \\ 0.293 & 0.905\end{array}\right)$
for
$|u\rangle=\left(\begin{array}{l}1 \\ 0\end{array}\right)$
$|w\rangle=\left(\begin{array}{l}\cos (2 \pi / 5) \\ \sin (2 \pi / 5)\end{array}\right)$.

Validities are computed via the trace formula:

$$
\begin{aligned}
\omega \vDash \operatorname{fem} \stackrel{(3)}{=} \operatorname{tr}(\omega \mathrm{fem})=\operatorname{tr}(|v\rangle\langle v \| u\rangle\langle u|) & =\operatorname{tr}(\langle u \mid v\rangle\langle v \mid u\rangle) \\
& =\operatorname{tr}(\overline{\langle v \mid u\rangle}\langle v \mid u\rangle) \\
& =\operatorname{tr}\left(|\langle v \mid u\rangle|^{2}\right) \\
& =|\langle v \mid u\rangle|^{2} \\
& =0.976 .
\end{aligned}
$$

As an aside, this same validity can be obtained as squared length of the projected vector fem $|v\rangle$, where $\omega=|v\rangle\langle v|$, since we are dealing with vector states and predicates:

$$
\begin{aligned}
\left.\| \text { fem }|v\rangle \|^{2}=\langle\text { fem } \mid v\rangle \mid \text { fem }|v\rangle\right\rangle & =\langle\mid u\rangle\langle u \mid v\rangle|| u\rangle\langle u \mid v\rangle\rangle \\
& =|\langle u \mid v\rangle|^{2} \cdot\langle u \mid u\rangle \\
& =|\langle v \mid u\rangle|^{2} .
\end{aligned}
$$

Similarly we get:

$\omega \vDash \operatorname{btr}=0.024$.

These validities in themselves are not very interesting. For the Linda example it is more relevant to compare them to the validities of the (sequential) conjunction $\&$ and disjunction | of these two predicates:

$$
\begin{aligned}
& \omega \vDash \text { fem } \& \text { btr } \stackrel{(1)}{=} \operatorname{tr}(\omega \sqrt{\text { fem }} \text { btr } \sqrt{\text { fem }}) \\
&= \operatorname{tr}(\omega \text { fem btr fem }) \\
& \text { since fem is a projection } \\
&= \operatorname{tr}(|v\rangle\langle v \| u\rangle\langle u|| w\rangle\langle w \| u\rangle\langle u|) \\
&= \operatorname{tr}\left(|\langle v \mid u\rangle|^{2} \cdot|\langle w \mid u\rangle|^{2}\right) \\
&=|\langle v \mid u\rangle|^{2} \cdot|\langle w \mid u\rangle|^{2} \\
&= 0.09315 .
\end{aligned}
$$

Notice that this computation can be simplified because the feminist predicate is a projection (idempotent), so that it equals its own square root: fem $=\sqrt{\text { fem. }}$. For more general predicates, that is, for effects instead of projections, these square roots remain. In a similar manner one computes:

$$
\omega \vDash \operatorname{btr} \mid \text { fem } \stackrel{(2)}{=} \omega \vDash \sim(\sim \text { btr } \& \sim \text { fem })=0.906 \text {. }
$$

We see that the probability that Linda is both a feminist and a bankteller (option (iii)) is higher than the probability that she is only a bankteller (option (ii)). Similarly, the probability that Linda is a bankteller or a feminist (option (iv)) is lower than the probability that she is just a feminist (option (i)).

Finally, we demonstrate that sequential conjunction \& and disjunction | are really non-commutative, and do not lead to fallacies in their second arguments:

$$
\begin{aligned}
\omega \vDash \operatorname{btr} \& \text { fem } & =0.00233 & \omega \vDash \text { fem } \mid \text { btr } & =0.998 \\
& \leq 0.976 & & \geq 0.024 \\
& =\omega \vDash \text { fem } & & =\omega \vDash \text { btr. }
\end{aligned}
$$

Quantum descriptions of the Linda example have been given earlier by several authors, see for instance Aerts (2009), Busemeyer, Pothos, Franco, and Trueblood (2011), Franco (2009) and Khrennikov (2010), in terms of successive applications of projections on a Hilbert space. Later on, in Eq. (6), we show that these successive projections correspond to sequential conjunction \&, as used in the above description. 
Remark 1. The above probabilities in the Linda example coincide with the numbers of Busemeyer and Bruza (2012). There is an important difference however: here these probabilities are obtained in an intuitive, high-level manner, via validities of generally defined 'and' and 'or' formulas in the logic of effects. In loc. cit. they arise from detailed low-level calculations via inner products in the Hilbert space $\mathbb{C}^{2}$, see the two points below. As illustrated in Appendix, via the EfProb library, all that complexity can remain hidden so that one can concentrate on the logical formulation. This logical approach is a stepping stone towards a formal symbolic logic for (quantum) probability, with a proof theory for deriving valid proposition.

We give a more detailed comparison of the different calculations, for the conjunction and disjunction fallacies separately.

(1) In the above example we explicitly formulate the predicate 'fem \& btr' and computed its validity. In contrast, Busemeyer and Bruza (2012, §4.1.1.2) only compute validities, but the conjunction predicate itself is not made explicit. Hence it is less clear what is going on, from a logical perspective. Using our notation, the validity of the conjunction predicate 'fem \& btr' is computed by Busemeyer and Bruza (2012) as $\|$ fem $|v\rangle\left\|^{2} \cdot\right\| \operatorname{btr}|u\rangle \|^{2}$. The underlying idea seems to be that the validity of conjunction predicates can be computed via Bayes' rule, see Eq. (8), namely as product $(\omega \vDash$ fem $) \cdot\left(\left.\omega\right|_{\text {fem }} \vDash\right.$ btr) .

(2) The literal formulation of the disjunction statement of Busemeyer and Bruza $(2012, \S 4.1)$ is: Linda is ... "active in the feminist movement or a bank teller". But in their computation, they put the bank teller first; Busemeyer and Bruza (2012, §4.1.1.3) explain the computation of the probability of the disjunction statement in words as: "Note that the probability of 'yes' to the disjunction equals the probability that 'no' to the bank teller and then 'no' to the feminist question does not occur", precisely as in the De Morgan formula for disjunction | in (2). This different order, with bank teller first, is indeed the only way to get a disjunction fallacy, since | is nonmonotone only in its first argument, see in (4) above. Such changes of the order of arguments are dangerous in a noncommutative setting. Being able to calculate the probability of a logical predicate like 'btr | fem' in a compositional manner helps to prevent them.

\section{Predicates as effects}

This section briefly explains the mathematics behind effects. It starts with the notions of 'effect algebra' and 'effect module', and illustrates how they arise naturally in probabilistic logic, both classical and quantum. More information and references can be found in Jacobs (2015).

We start with the notion of partial commutative monoid. It is given by a set $M$ with partial binary sum operation $\otimes$ and a neutral element 0 for $\varnothing$, so that $x \oslash 0=x$ for all $x \in M$. The commutativity and associativity requirements for $\otimes$ hold in a suitable partial sense. The partiality of the sum may look a bit strange at first, but arises naturally, for instance in the unit interval $[0,1]$, where the sum of two element $x, y \in[0,1]$ does not always exist.

A effect algebra is a partial commutative monoid in which each element $x$ has a unique orthosupplement $\sim x$, sometimes written as $x^{\perp}$, with $x \oslash \sim x=1$, where $1=\sim 0$. There is an additional technical requirement that 0 is the only element $x$ for which $x \otimes$ 1 is defined. Next, an effect module is an effect algebra with a scalar multiplication $r \cdot x$, for $r \in[0,1]$, satisfying some obvious properties. We present the main examples.

\section{Example 2.}

(1) Let $X$ be an arbitrary set. A 'fuzzy' predicate on $X$ is a function $p: X \rightarrow[0,1]$, giving for each element $x \in X$ its 'degree of truth' $p(x) \in[0,1]$. Such a predicate is called sharp if either $p(x)=0$ or $p(x)=1$ for all $x$, that is, if $p$ restricts to $X \rightarrow\{0,1\}$; it then corresponds to a subset $U \subseteq X$, via the indicator function $\mathbf{1}_{U}: X \rightarrow\{0,1\}$, with $\mathbf{1}_{U}(x)=1$ iff $x \in U$.

As is well-known, sharp predicates form a Boolean algebra-and thereby also an effect algebra. What is less familiar is that fuzzy predicates on a set $X$ form an effect module. First, there is a partial sum $p \otimes q$ of $p, q: X \rightarrow[0,1]$ which is defined if $p(x)+q(x) \leq 1$ for all $x \in X$; in that case $(p \otimes q)(x)=p(x)+q(x)$. The function $\mathbf{0}: X \rightarrow[0,1]$, given by $\mathbf{0}(x)=0$, is neutral element for $\otimes$. The orthosupplement $\sim p: X \rightarrow[0,1]$ is defined as $(\sim p)(x)=1-p(x)$. Clearly, $p \otimes \sim p=\mathbf{1}$, where $\mathbf{1}=\sim \mathbf{0}$ is the constant-one predicate. This means that the set $[0,1]^{X}$ of fuzzy predicates on $X$ is an effect algebra.

Fuzzy predicates, but not the sharp ones, are closed under scalar multiplication. For $p: X \rightarrow[0,1]$ and $r \in[0,1]$ we define $r \cdot p: X \rightarrow[0,1]$ as $(r \cdot p)(x)=r \cdot p(x)$. Thus, $r \cdot p$ is a 'scaled' version of $p$.

(2) There is an analogous story in the quantum case. Let $\mathscr{H}$ be a Hilbert space. A predicate on $\mathscr{H}$ is a bounded linear operator $p: \mathscr{H} \rightarrow \mathscr{H}$ with $0 \leq p \leq$ id. Such predicates are often called effects. A predicate/effect is called sharp if it is a projection, that is, if $p^{2}=p$, where $p^{2}=p p$ is the composition of $p$ with itself. It is a standard result that an operator $p$ on a finite-dimensional Hilbert space is an effect if and only if all its eigenvalues are in the unit interval $[0,1]$. Similarly, $p$ is a projection if and only if its eigenvalues are either 0 or 1 , that is, are in $\{0,1\} \subseteq[0,1]$.

Since Birkhoff and von Neumann (1936) it is known that projections form an orthomodular lattice (Kalmbach, 1983)and thus also an effect algebra. It is again less well-known that (non-sharp) predicates form an effect module. The sum of two predicates $p \otimes q$ is defined if the point-wise sum $p+q$ of operators satisfies $p+q \leq \mathrm{id}$, and in that case $p \otimes q$ equals $p+q$. The orthosupplement $\sim p$ is id $-p$. The falsity predicate 0 : $\mathscr{H} \rightarrow \mathscr{H}$ is the constant function, mapping everything to 0 . Its orthosupplement $\mathbf{1}=\sim \mathbf{0}=\mathrm{id}-\mathbf{0}=$ id is the identity operator $\mathscr{H} \rightarrow \mathscr{H}$.

Effects are closed under scalar multiplication, but projections are not. Indeed, for an effect $p: \mathscr{H} \rightarrow \mathscr{H}$ and a scalar $r \in[0,1]$, the point-wise multiplication $r \cdot p$ is again an effect.

Scalar multiplication of predicates can be used to express the relative influence of factors, for instance in a (simplified) medical context ${ }^{3}$ where the occurrence of a cough can be explained from either a cold or lung disease, via a predicate definition with a disjunction |, and with weights as scalars:

cough $=0.7 \cdot$ cold $\mid 0.2 \cdot$ long-disease.

Effects on a Hilbert space are the essential ingredients of positiveoperator valued measures (POVMs), which are used in quantum measurement. In essence, such a POVM is given by a collection of operators $E_{i}: \mathscr{H} \rightarrow \mathscr{H}$ satisfying $E_{i} \geq 0$ and $\sum_{i} E_{i}=$ id. This implies $E_{i} \leq$ id, so that each $E_{i}$ is an effect. A collection of such effects adding up to the identity (truth) is often called a test. Each single effect $E$ forms a 2-element POVM/test $E, E^{\perp}$.

The effect modules of fuzzy predicates on a set, and of effects on a Hilbert space, as described in Example 2, have another logical

\footnotetext{
3 Such examples are used for instance in the online book: N. Goodman and J Tenenbaum, Probabilistic Models of Cognition at https://probmods.org/.
} 
operation, namely sequential conjunction \&. For fuzzy predicates it is defined by point-wise multiplication:

$(p \& q)(x)=p(x) \cdot q(x)$.

This operation \& with the truth predicate $\mathbf{1}$ forms a monoid on the set $[0,1]^{X}$ of fuzzy predicates. It is a commutative monoid, since $p \& q=q \& p$. This conjunction preserves the order (is monotone) in both arguments, so that $p \& q \leq p \& \mathbf{1}=p$ and similarly $p \& q \leq q$. Hence conjunction fallacies are impossible with this conjunction; similarly, disjunction fallacies do not occur with the associated disjunction, via (2). Sequential conjunction restricts to intersection when applied to sharp predicates, given by indicator functions: $\mathbf{1}_{U} \& \mathbf{1}_{V}=\mathbf{1}_{U \cap V}$. Notice that $p \in[0,1]^{X}$ is sharp iff $p \& p=p$.

In the quantum case there is also a sequential conjunction operation $\&$, via $p \& q=\sqrt{p} q \sqrt{q}$ for effects $p, q$, as already described in Eq. (1). It has truth $\mathbf{1}$ as neutral element in both arguments. This operation \& is not commutative, as illustrated in Eq. (4) above. Moreover, \& does preserve the order in its second argument, but not in its first argument. Hence one always has $p \& q \leq p \& \mathbf{1}=$ $p$, but inequalities $p \& q \geq q$ may occur-giving a conjunction fallacy as in Example 1. Via the 'De Morgan' definition of sequential disjunction | we can thus also have inequalities $q \geq p \mid q$, forming a disjunction fallacy.

There are several things worth noticing about (quantum) sequential conjunction \& and projections (sharp predicates).

(1) Jacobs (2015) shows that an effect $p$ is sharp if and only if it is idempotent, that is, if and only if $p \& p=p$. As mentioned above, this equivalence also holds for fuzzy predicates.

(2) Projections are not closed under \&. Explicitly, if $p$ and $q$ are projections, then $p \& q$ need not be a projection. This is shown explicitly in (9), below, using the previous point. But for instance, the 'bankteller and-then feminist' predicate btr \& fem, used in Example 1, is also not a projection, even though both btr and fem are projections.

(3) The conjunction formula $p \& q=\sqrt{p} q \sqrt{p}$ from Eq. (1) is well-established, going back to Gudder and Greechie (2002), but it lacks a clear intuition-like so many other phenomena in quantum theory. Gudder and Greechie (2002) speak of “a sequential product with natural properties" and believe that its properties are "physically motivated and can be tested, for example, in the optical bench situation", as in Example 3 below. We would like to add two formal arguments in support of the quantum conjunction formula $p \& q=\sqrt{p} q \sqrt{p}$.

- In the general context of effectus theory (Cho et al., 2015; Jacobs, 2015) it is shown that quantum sequential conjunction satisfies the same abstract properties as probabilistic and Boolean conjunction.

- When the predicates $p, q$ are restricted to projections, the validity of $p \& q$ coincides with the traditional understanding of the validity of conjunction in a vector state $\omega=|v\rangle\langle v|$, in terms of successive applications of projective operators:

$$
\begin{aligned}
\omega \vDash p \& q & =\operatorname{tr}(\omega \sqrt{p} q \sqrt{p}) \\
& =\operatorname{tr}(|v\rangle\langle v| p q q p) \\
& =\operatorname{tr}(\langle v|p q q p| v\rangle) \\
& =\langle v|p q q p| v\rangle \\
& =\langle q p \mid v\rangle|q p| v\rangle\rangle \\
& =\| q p|v\rangle \|^{2} .
\end{aligned}
$$

We see that effects form a logically richer class of predicates than projections, since they are closed under scalar multiplication and under sequential conjunction. Later, in Section 5, we shall see that they are also closed under predicate transformation w.r.t. a channel. All this show that several useful logical constructions do not exist within the class of projections.

\section{Conditioning of states}

This section shows how a conditional state $\left.\omega\right|_{p}$ can be obtained from a state $\omega$ and a predicate $p$. It is pronounced as " $\omega$ given $p$ " and is understood as an update (or revision) of the state $\omega$ in the light of the information provided by the predicate $p$. This conditioning of states is very natural in Bayesian probability. It can be defined uniformly in effectus theory, both for the quantum and classical (non-quantum) case, but conditioning is usually not an explicit operation in quantum theory.

Conditioning of states is a partial operation. For a state $\omega$ and predicate $p$ on the same Hilbert space, the conditional state $\left.\omega\right|_{p}$ is defined only if the validity $\omega \vDash p$ is non-zero. In that case one defines:

$\left.\omega\right|_{p}=\frac{\sqrt{p} \omega \sqrt{p}}{\omega \vDash p}$.

There is a close connection with Bayes' rule, which takes the following form:

$$
\left.\omega\right|_{p} \vDash q=\frac{\omega \vDash p \& q}{\omega \vDash p} .
$$

This rule holds in a quantum setting, where the order of $p$ and $q$ is important in sequential conjunction $p \& q$, because of noncommutativity. But the rule of total probability fails in the quantum world, see at the end of Example 3 below.

Another important property of conditioning is that the order is relevant, that is, $\left.\left(\left.\omega\right|_{p}\right)\right|_{q}$ is in general not the same as $\left.\left(\left.\omega\right|_{q}\right)\right|_{p}$. Later on in Example 5 the cognitive analogue of this fact will be illustrated via the relevance of the order of conditioning/priming. At this stage we present a simple illustration of the difference, building on Example 1.

$$
\left.\left(\left.\omega\right|_{\text {fem }}\right)\right|_{\text {btr }} \vDash \text { fem }=\left.0.0955\left(\left.\omega\right|_{\text {btr }}\right)\right|_{\text {fem }} \vDash \text { fem }=1 .
$$

There is a special case of the conditioning formula (7) that is worth making explicit. When the state $\omega$ is given as a vector state and the predicate $p$ is a projection, then the conditioned state $\left.\omega\right|_{p}$ is again a vector state. This works as follows. Let $\omega=|v\rangle\langle v|$ and $p$ be a projection so that $\sqrt{p}=p$ and $p^{\dagger}=p$. Then:

$$
\left.\omega\right|_{p} \stackrel{(7)}{=} \frac{p|v\rangle\langle v| p}{\omega \vDash p}=|w\rangle\langle w| \quad \text { for }|w\rangle=\frac{1}{\sqrt{\omega \vDash p}} p|v\rangle .
$$

But in general, the conditioned state $\left.\omega\right|_{p}$ need not be a vector state.

We illustrate conditioning of states via a standard example from physics.

Example 3. Polarised light filters are a great illustration of the 'weirdness' of the quantum world. Briefly, if we have a source $v$ emitting vertically polarised light we can check how much light goes through polarised filters:

- if we use a vertical filter, indicated as $\uparrow$, all the light goes through;

- when we use a horizontal filter $\leftrightarrow$, all light is blocked.

But if we put a diagonally oriented filter $\measuredangle$ in between $\uparrow$ and $\leftrightarrow$, light does go through the sequence of filters $\uparrow, \nearrow, \leftrightarrow$, namely one quarter of it. Here we describe this situation via sequential conjunction and conditioning of states, following Jacobs (2015). For more background information see e.g. Rieffel and Polak (2011).

We start with a state $v$ capturing a vertically polarised light source, together with three idempotent (projection) predicates for 'vertical', 'horizontal' and 'diagonal' filters.

$$
v=\left(\begin{array}{ll}
0 & 0 \\
0 & 1
\end{array}\right) \quad \uparrow=\left(\begin{array}{ll}
0 & 0 \\
0 & 1
\end{array}\right) \leftrightarrow=\left(\begin{array}{ll}
1 & 0 \\
0 & 0
\end{array}\right) \quad \swarrow=\frac{1}{2}\left(\begin{array}{ll}
1 & 1 \\
1 & 1
\end{array}\right)
$$


The following validities reflect which proportion of vertically polarised light comes through a filter, described as predicate, which is polarised respectively as $\uparrow, \leftrightarrow$, and $\nearrow$.

$\nu \vDash \uparrow=1 \quad \nu \vDash \leftrightarrow=0 \quad \nu \vDash \neg=\frac{1}{2}$.

Next we ask for the probability of vertically polarised light coming through if we first use a diagonal filter "and then" a horizontal one.

$v \vDash \nearrow \quad \& \leftrightarrow=\frac{1}{4} \quad$ or, more explicitly, $v \vDash \downarrow \&(\nearrow \& \leftrightarrow)=\frac{1}{4}$.

Now one quarter is horizontally polarised! If we do this in the other order, we see nothing:

$v \vDash \leftrightarrow \& \swarrow^{\nearrow}=0$.

We emphasise that by using these conjunctions \& our predicates are no longer projections, that is, idempotent:

$$
\begin{aligned}
\leftrightarrow \& \swarrow= & \frac{1}{4}\left(\begin{array}{ll}
1 & 1 \\
1 & 1
\end{array}\right) \text { whereas }(\leftrightarrow \& \swarrow) \& \\
& (\leftrightarrow \& \swarrow)=\frac{1}{8}\left(\begin{array}{ll}
1 & 1 \\
1 & 1
\end{array}\right) .
\end{aligned}
$$

If we enforce diagonal polarisation via conditioning and then ask how much of the light is horizontally polarised we get a probability of one half. Since this is the first time where we see a state update, we expand the calculation.

$$
\begin{aligned}
& \left.\nu\right|_{\swarrow} \vDash \leftrightarrow \stackrel{(7)}{=} \frac{1}{v \vDash \nearrow} \operatorname{tr}(\sqrt{\nearrow} v \sqrt{\nearrow} \leftrightarrow) \\
& =\frac{1}{1 / 2} \operatorname{tr}(\nearrow \vee \swarrow \leftrightarrow) \\
& =2 \operatorname{tr}\left(\frac{1}{4}\left(\begin{array}{ll}
1 & 1 \\
1 & 1
\end{array}\right)\left(\begin{array}{ll}
0 & 0 \\
0 & 1
\end{array}\right)\left(\begin{array}{ll}
1 & 1 \\
1 & 1
\end{array}\right)\left(\begin{array}{ll}
1 & 0 \\
0 & 0
\end{array}\right)\right) \\
& =\frac{1}{2} \operatorname{tr}\left(\left(\begin{array}{ll}
0 & 1 \\
0 & 1
\end{array}\right)\left(\begin{array}{ll}
1 & 1 \\
1 & 1
\end{array}\right)\left(\begin{array}{ll}
1 & 0 \\
0 & 0
\end{array}\right)\right) \\
& =\frac{1}{2} \operatorname{tr}\left(\left(\begin{array}{ll}
1 & 1 \\
1 & 1
\end{array}\right)\left(\begin{array}{ll}
1 & 0 \\
0 & 0
\end{array}\right)\right) \\
& =\frac{1}{2} \operatorname{tr}\left(\begin{array}{ll}
1 & 0 \\
1 & 0
\end{array}\right) \\
& =\frac{1}{2} \text {. }
\end{aligned}
$$

This polarisation example can also be used to illustrate the failure of the law of total probability in the quantum setting. We already know that the probability $v \vDash \leftrightarrow$ is zero. But:

$$
\left(\left.v\right|_{\swarrow^{\nearrow}} \vDash \leftrightarrow\right) \cdot\left(v \vDash \swarrow^{\prime}\right)+\left(\left.v\right|_{\sim_{\nearrow^{\nearrow}} \vDash \leftrightarrow}\right) \cdot\left(v \vDash \sim_{\swarrow^{\nearrow}}\right)=\frac{1}{2} .
$$

Since Bayes' rule (8) does hold in the quantum world, the latter probability can also be obtained as:

$$
\begin{aligned}
(v \vDash \nearrow \quad \& \leftrightarrow)+(v \vDash \sim \nearrow \& \leftrightarrow) & =v \vDash(\nearrow \& \leftrightarrow)+(\sim \nearrow \& \leftrightarrow) \\
& =\frac{1}{2} .
\end{aligned}
$$

This example can also be used to see that sequential conjunction $\&$ is not only non-commutative but also non-associative:

$$
v \vDash \uparrow \&(\nearrow \& \leftrightarrow)=\frac{1}{4} \quad \text { but } v \vDash(\uparrow \& \measuredangle) \& \leftrightarrow=0 .
$$

Remark 2. Conditioning is a basic operation in classical, i.e. nonquantum, probability and plays a fundamental role in Bayesian reasoning. It involves a probability distribution (or measure) $\omega$ and an event $A$, and yields a new distribution $\omega(-\mid A)$-which we prefer to write here as $\left.\omega\right|_{A}$. It is an update (revision) of the original distribution $\omega$, given event $A$, that is, in the light of the evidence $A$. This only works if the event $A$ itself has non-zero probability.

Events $A$ are often identified with subsets, that is, with sharp $\{0,1\}$-valued predicates, as described in Example $2(1)$. The whole area of fuzzy logic however, advocates that predicates are more naturally understood as $[0,1]$-valued functions, capturing degrees of truth. The integration of such fuzzy logic and probability goes back to Zadeh (1968), whose formulas we follow for classical probabilistic/fuzzy logic, e.g. for conjunction \& via point-wise multiplication (5), but also for conditioning. In order to emphasise the similarity with the quantum description of conditioning (7) that we use in this section, we briefly mention the essentials of the fuzzy approach from Zadeh (1968), in the discrete case: a state on a set $X$ is identified with a finite discrete probability distribution on $X$, given by a probability mass function $\omega: X \rightarrow[0,1]$ with finite support and with $\sum_{x} \omega(x)=1$. The validity (or expected value) $\omega \vDash p$ of a fuzzy predicate $p \in[0,1]^{X}$ in state $\omega$ is given by $\sum_{x} \omega(x) \cdot p(x)$. If this validity $\omega \vDash p$ is non-zero, then we can define the updated state $\left.\omega\right|_{p}=\omega(-\mid p): X \rightarrow[0,1]$ on $X$ as normalised product:

$\left.\omega\right|_{p}(x)=\frac{\omega(x) \cdot p(x)}{\omega \vDash p}$.

Bayes' rule then holds, precisely in the form (8). In fuzzy logic the order of conditioning does not matter, since: $\left.\left(\left.\omega\right|_{p}\right)\right|_{q}=\left.\omega\right|_{p \& q}=$ $\left.\omega\right|_{q \& p}=\left.\left(\left.\omega\right|_{q}\right)\right|_{p}$.

\section{Channels, for state and predicate transformation}

Let $\mathscr{H}$ and $\mathscr{K}$ be two (finite-dimensional, complex) Hilbert spaces. A channel $c$ from $\mathscr{H}$ to $\mathscr{K}$ is a function, $\mathscr{B}(\mathscr{K}) \rightarrow \mathscr{B}(\mathscr{H})$ in the opposite direction between the associated spaces of operators $\mathscr{B}(\mathscr{K})$ and $\mathscr{B}(\mathscr{K})$ on $\mathscr{K}$ and $\mathscr{H}$, which is linear, completely positive and unital. We refer to Nielsen and Chuang $(2000, \S 8.2)$ for the mathematical details. All we need here is that there are two basic transformation operations associated with a channel, namely state transformation $c \gg \omega$ and predicate transformation $c \ll q$. These operations work in opposite direction: if $\omega$ is a state of $\mathscr{H}$, then $c \gg \omega$ is a state of $\mathscr{K}$. And if $q$ is a predicate on $\mathscr{K}$, then $c \ll$ $q$ is a predicate on $\mathscr{H}$. These transformations are related by the fundamental transformation validity equation:

$(c \gg \omega) \vDash q=\omega \vDash(c \ll q)$.

Channels are extremely useful in quantum logic, as will be illustrated below. They do not work well in the Birkhoff-von Neumann logic using projections as predicates, since predicate transformation $c \ll q$ need not produce a projection when $q$ is a projection. In contrast, effects are closed under this predicate transformation. This allows us to move states and predicates from one Hilbert space to another.

There are several ways to construct channels. In the example below we obtain a channel from a unitary matrix $U$, via the standard construction $U(-) U^{\dagger}$. Channels of this special 'pure' form do preserve projections. But the matrix $U$ need not be unitary to produce a channel in this way: it suffices that $U$ is an isometry. In fact, there is a general 'Kraus' representation of channels, saying that each channel can be written of the form $\sum_{i} U_{i}(-) U_{i}^{\dagger}$, where $\sum_{i} U_{i}^{\dagger} U_{i}=$ id, see Nielsen and Chuang (2000) for details. In that general form, projections are not closed under predicate transformation. As simple example, with a single isometric $3 \times 2$ matrix, consider:

$V=\left(\begin{array}{ll}0 & 0 \\ 1 & 0 \\ 0 & 1\end{array}\right) \quad$ with $\quad V^{\dagger}=\left(\begin{array}{lll}0 & 1 & 0 \\ 0 & 0 & 1\end{array}\right) \quad$ and $\quad V^{\dagger} V=$ id.

Then $V(-) V^{\dagger}$ is a channel, that can transform states on $\mathbb{C}^{2}$ into states on $\mathbb{C}^{3}$, and, in the other direction, can transform predicates on $\mathbb{C}^{3}$ to predicates on $\mathbb{C}^{2}$. Channels can also be used for projecting (marginalisation) and for measurement, see Nielsen and Chuang (2000), but also Jacobs and Cho (2017). Channels given by unitaries form a proper subclass of reversible channels.

Example 4. We illustrate how the quantum setting can be used to capture different personal perspectives or contexts, via different states. The example is copied again from Busemeyer and Bruza (2012); we use the same vectors (states) and obtain the same probabilities, but via a slightly different description, using a channel. 
A couple consisting of a man $M$ and a woman $W$ wish to buy a new car together. They consider three brands, namely Audi $(A)$, BMW $(B)$, and Cadillac $(C)$, which will be modelled as the three basic predicates, with domain of size 3, obtained from the orthonormal basis vectors $\left|e_{1}\right\rangle=\left(\begin{array}{l}1 \\ 0 \\ 0\end{array}\right),\left|e_{2}\right\rangle=\left(\begin{array}{l}0 \\ 1 \\ 0\end{array}\right),\left|e_{3}\right\rangle=\left(\begin{array}{l}0 \\ 0 \\ 1\end{array}\right)$ as:

$$
\begin{array}{ll}
A=\left|e_{1}\right\rangle\left\langle e_{1}\left|=\left(\begin{array}{lll}
1 & 0 & 0 \\
0 & 0 & 0 \\
0 & 0 & 0
\end{array}\right) \quad B=\right| e_{2}\right\rangle\left\langle e_{2}\right|=\left(\begin{array}{lll}
0 & 0 & 0 \\
0 & 1 & 0 \\
0 & 0 & 0
\end{array}\right) \\
C=\left|e_{3}\right\rangle\left\langle e_{3}\right|=\left(\begin{array}{lll}
0 & 0 & 0 \\
0 & 0 & 0 \\
0 & 0 & 1
\end{array}\right) .
\end{array}
$$

Clearly, these three predicates form a test: they satisfy $A+B+C=$ 1.

The man's perspective is captured via a particular vector state:

$$
M=|v\rangle\langle v| \quad \text { for } \quad|v\rangle=\left(\begin{array}{l}
-0.6963 \\
0.6963 \\
0.1741
\end{array}\right) \in \mathbb{C}^{3} .
$$

This leads to the following validities, interpreted as the man's preferences for the individual car brands.

$$
M \vDash A=0.485 \quad M \vDash B=0.485 \quad M \vDash C=0.030 .
$$

The woman's perspective is described via a different state $W$. It can be defined via state transformation $W=c h \gg M$ from the man's state $M$, where the channel $c h$ is determined by a unitary matrix $U$, so that $c h \gg M=U^{\dagger} M U$, where $U^{\dagger}$ is the conjugate transpose. The matrix $U$ is copied from Busemeyer and Bruza (2012):

$U=\left(\begin{array}{lll}1 / \sqrt{2} & 1 / 2 & -1 / 2 \\ 1 / \sqrt{2} & -1 / 2 & 1 / 2 \\ 0 & 1 / \sqrt{2} & 1 / \sqrt{2}\end{array}\right)$.

The woman's preferences are then given by the following validities.

$W \vDash A=0 \quad W \vDash B=0.329 \quad W \vDash C=0.671$.

Via the transformation validity Eq. (10) we can also obtain the latter preferences of the woman in the man's state as validities of the transformed predicates:

$$
\begin{aligned}
& M \vDash c h \ll A=0 \\
& M \vDash c h \ll C=0.671 .
\end{aligned}
$$

Thus, via these predicate transformations $c h \ll(-)=U(-) U^{\dagger}$ the man can see the woman's perspective in his own state. In the remainder of this example we shall use these transformed predicates for the woman's preferences.

We can now describe 'order effects', like in Example 3 on polarisation. The man's preference for a BMW, when he first evaluates the woman's preference for a Cadillac, is given by:

$$
M \vDash(c h \ll C) \& B=0.168 \text {. }
$$

Interestingly, taking the woman's preference for a Cadillac into account, the man's preference for a BMW goes down, w.r.t. the value $M \vDash B=0.485$ given above in (13). But if the man evaluates the woman's preference for a Cadillac first, his own preference for the Cadillac goes up, a little bit, wrt. the value of $M \vDash C$ in (13):

$M \vDash($ ch $\ll C) \& C=0.336$.

This is another 'conjunction fallacy' example, where a conjunction has a higher probability than its second components, like in Example 1 . That does not happen in classical probabilistic logic, as discussed in Section 3.

Let us now assume that the man and woman focus on making the same choice. They do so by looking at their joint state $M \otimes W$, obtained as Kronecker product, and conditioning it to the situation where their choices coincide. We abbreviate this updated joint state as $\omega$ in:

$\omega=\left.(M \otimes W)\right|_{A \otimes A|B \otimes B| C \otimes C}$.

The tensor of predicates $A \otimes A$ is 'parallel conjunction', also given by the Kronecker product. We then compute the probabilities for the various brands:

$\omega \vDash A \otimes A=0 \quad \omega \vDash B \otimes B=0.887 \quad \omega \vDash C \otimes C=0.113$.

Hence it is most likely that they buy a BMW together. It is not hard to see that these three probabilities arise via normalisation from the three numbers:

$$
(M \vDash A) \cdot(W \vDash A) \quad(M \vDash B) \cdot(W \vDash B) \quad(M \vDash C) \cdot(W \vDash C) .
$$

Finally, we take another look at the situation in this example. As shown above, the woman's preference for an Audi is zero. Hence we can describe her as stemming from a world with only two relevant brands, namely BMW and Cadillac. Thus we can re-define her as living in the 'smaller' world $\mathbb{C}^{2}$, instead of $\mathbb{C}^{3}$. The relevant numbers actually already occur in Busemeyer and Bruza (2012):

$$
W_{2}=|w\rangle\langle w| \quad \text { for } \quad|w\rangle=\left(\begin{array}{l}
-0.5732 \\
0.8194
\end{array}\right) \in \mathbb{C}^{2} .
$$

We now use the channel $d$ associated with the isometry $V: \mathbb{C}^{2} \rightarrow$ $\mathbb{C}^{3}$ in (11) to transform the above state $W_{2}$ on $\mathbb{C}^{2}$ into a state $W_{3}=d \gg W_{2}$ on $\mathbb{C}^{3}$. The numbers of $|w\rangle$ are chosen in such a way that, as before:

$W_{3} \vDash A=0 \quad W_{3} \vDash B=0.329 \quad W_{3} \vDash C=0.671$.

As an aside, the woman's vector $|w\rangle \in \mathbb{C}^{2}$ can also be obtained as $V^{\dagger} U^{\dagger}|v\rangle$, where $|v\rangle \in \mathbb{C}^{3}$ is the vector used for the man's state, from (12).

\section{Order inference effects}

As observed in the beginning of Section 4, there is in general a difference between the order of conditioning of states $\left.\left(\left.\omega\right|_{p}\right)\right|_{q}$ and $\left.\left(\left.\omega\right|_{q}\right)\right|_{p}$ in quantum logic. This means that the order in which evidence, given by the predicates $p, q$, is presented has influence on the resulting (multiple times conditioned) state. This phenomenon has been picked up in the area of quantum cognition, where it is used to explain what is known as the 'order inference effect' in psychology: the order in which people are primed with evidence is relevant for what they believe. This is illustrated in the following example, which comes in essence from Busemeyer and Bruza (2012), but is slightly adapted at the end.

Example 5. Consider a court case, with three points of view: the prosecutor's perspective $P$, the defence's perspective $D$, and the judge's perspective $J$. These three perspectives are modelled as three states $P, D, J$, each with four dimensions. They are connected via unitary matrices - actually via the associated channels - as suggested in:

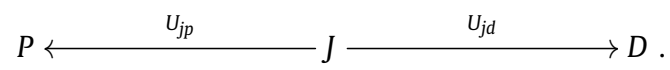

The precise form of these unitary matrices does not matter too much, but we copy the details from the original source. ${ }^{4}$ First, one starts from a self-adjoint matrix $H$, which is used as 'Hamiltonian', like in the solution of Schrödinger's (time-independent)

\footnotetext{
4 The Hamiltonian matrix $H$ that we use here is defined by Busemeyer and Bruza (2012, §4.2.4.1) as sum $H=H_{1}+H_{2}$, where $H_{1}$ contains a typo in one matrix entry.
} It is corrected here. 
wave equation. It is used to form the above two unitary matrices $U_{j p}$ and $U_{j d}$ via a matrix-exponent:

$H=\left(\begin{array}{llll}2 & 1 & 1 & 0 \\ 1 & -2 & 0 & 1 \\ 1 & 0 & 0 & 1 \\ 0 & 1 & 1 & 0\end{array}\right) \quad$ with $U_{j p}=e^{1.2393 \cdot i \cdot H} \quad U_{j d}=e^{-3.8324 \cdot i \cdot H}$.

We write $c h_{j p}$ and $c h_{j d}$ for the channels induced by the unitaries $U_{j p}$ and $U_{j d}$, as in Example 4.

With this infrastructure in place, we can define the three states, corresponding to the three different perspectives.

$J=|v\rangle\langle v|$ for $|v\rangle=\left(\begin{array}{l}\sqrt{0.459 / 2} \\ \sqrt{0.459 / 2} \\ \sqrt{0.541 / 2} \\ \sqrt{0.541 / 2}\end{array}\right)$ and $\left\{\begin{array}{l}P=c h_{j p} \gg J \\ D=c h_{j d} \gg J .\end{array}\right.$

Notice that the prosecutor and defence states $P$ and $D$ are obtained from the Judge's state $J$ via state transformation, using different channels.

The information that is presented in this court case is modelled by the four basic predicates on our 4-dimensional space. There are four kinds of information: positive/negative guilt-hypotheses and positive/negative evidence (w.r.t. guilt). They are captured by the following abbreviations.

$\mathrm{pGpE}=\left|e_{1}\right\rangle\left\langle e_{1}|\quad \mathrm{pGnE}=| e_{2}\right\rangle\left\langle e_{2}\right|$

$\mathrm{nGpE}=\left|e_{3}\right\rangle\left\langle e_{3}|\quad \mathrm{nGnE}=| e_{4}\right\rangle\left\langle e_{4}\right|$.

The $e_{i}$ are the standard basis vectors of $\mathbb{C}^{4}$. We now compute:

$J \vDash \mathrm{pGpE} \mid \mathrm{pGnE}=0.459 \quad$ and $\quad J \vDash \mathrm{nGpE} \mid \mathrm{nGnE}=0.541$.

We see that the judge has a slight bias towards innocence (nonguilt).

We turn to the prosecutor's perspective, in which guilt is seen as more likely:

$P \vDash \mathrm{pGpE} \mid \mathrm{pGnE}=0.834$.

Via predicate transformation these same probabilities can be obtained in the judge's state, using Eq. (10):

$J \vDash c h_{j p} \ll(\mathrm{pGpE} \mid \mathrm{pGnE})=0.834$.

Like in Example 4, such predicate transformation allows the judge to see someone else's perspective in his/her own state.

The defence is in this case apparently not really convinced of innocence:

$D \vDash \mathrm{pGpE} \mid \mathrm{pGnE}=0.660$.

The judge digests both the positive evidence $\mathrm{pGpE} \mid \mathrm{pGnE}$ of the prosecutor, via the channel $c h_{j p}$, and the negative evidence $\mathrm{pGnE}$ | $\mathrm{nGnE}$ of the defence, via the channel $c h_{j d}$. This evidence is collected in the two (transformed) predicates called $P_{e v}$, for prosecutor's evidence, and $D_{e v}$, for defence's evidence.

$P_{e v}=c h_{j p} \ll(\mathrm{pGpE} \mid \mathrm{nGpE}) \quad D_{e v}=c h_{j d} \ll(\mathrm{pGpE} \mid \mathrm{nGpE})$.

Subsequently we use these two predicates to condition the (state of the) judge, in two different orders. In each of the two conditioned states, the guilt question is asked. This yields probabilities:

$\left.\left(\left.J\right|_{P_{e v}}\right)\right|_{D_{e v}} \vDash \mathrm{pGpE} \mid \mathrm{pGnE}=0.29$

$\left.\left(\left.J\right|_{D_{e v}}\right)\right|_{P_{e v}} \vDash \mathrm{pGpE} \mid \mathrm{pGnE}=0.35$.

Hence the judge is most likely to acquit. But more importantly, the likelihood depends on the order in which the evidence is presented: a clear 'order inference effect'. The judge is most convinced by the one who speaks last. This is a very human characteristic.

\section{Conclusion}

We have sketched the essential aspects of modern quantum effect logic, involving states and predicates, validity and conditioning, and state and predicate transformation via channels. The use of these logical constructions has been demonstrated in several standard examples in quantum cognition. Moreover, the differences with the dominant, but more restricted, focus on projection predicates has been pointed out: effect predicates are closed under more logical operations - like sequential conjunction and disjunction, scaling, and predicate transformation along channels and thus provide a higher level of logical expressivity. Hence, the abstractness of quantum effect logic makes it easier to see and use the logical essentials directly, without getting bogged down in calculations with inner products and traces in Hilbert spaces that obscure the relevant logical operations. Moreover, via the implementation of the quantum effect logic in the EfProb library (in Python ) these effect predicates can be formulated naturally and their validity probabilities are computed easily, see also Appendix below. Hopefully all this will give a boost to the use of quantum logic in cognition research.

\section{Acknowledgments}

Thanks are due to Kenta Cho and to Aleks Kissinger for their feedback on a first version of this paper, and also to the reviewers for their helpful comments and questions.

\section{Appendix. Tool support for effect logic}

Effect logic has a firm theoretical foundation in effect modules, arising naturally in effectuses (Jacobs, 2015). In addition, it comes with practical tool support (Cho \& Jacobs, 2017) for the formalisation of states, predicates and channels, and for the calculation of validities $\omega \vDash p$ and updates $\left.\omega\right|_{p}$. This tool support takes the form of an embedding in the programming language Python, called EfProb, an abbreviation for Effectus Probability. ${ }^{5}$ It has been used for all the examples in this paper. In this appendix we give a sketch of how this works, for two of the earlier Examples, namely 1 and 4. In the code fragments below we use the interactive mode of Python, where user-input is preceded by $>>>$. The program's output, if any, is printed without preceding text.

In the Linda Example the relevant state and the predicates look as follows.

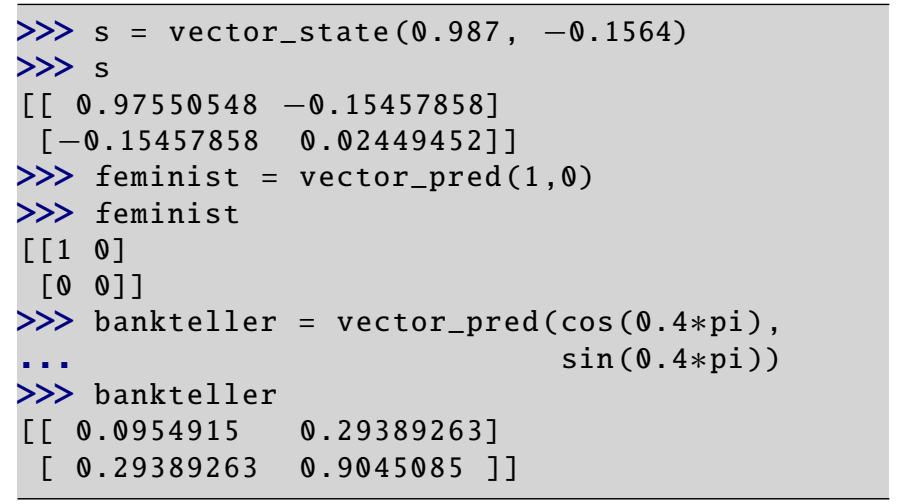

\footnotetext{
5 EfProb is publicly available via the website efprob.cs.ru.nl, together with an extensive user manual. All the EfProb code that is used in this article will be available
} there, in a separate file that can be executed on its own. 
Next we can compute validities. Since the mathematical validity sign $\vDash$ already has a fixed meaning Python, EfProb uses the notation $>=$ for $\vDash$, because it looks somewhat similar.

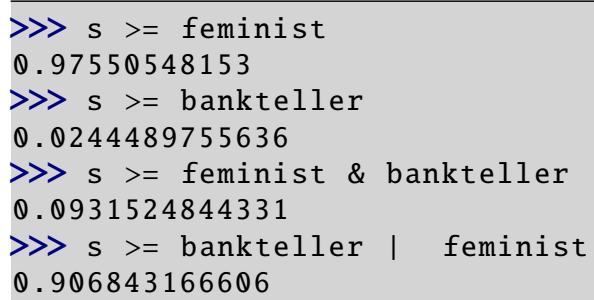

This demonstrates the conjunction and disjunction fallacies.

Next we describe the car preference Example 4 in EfProb. The car predicates are defined first, followed by the man's state and preferences, as validities.

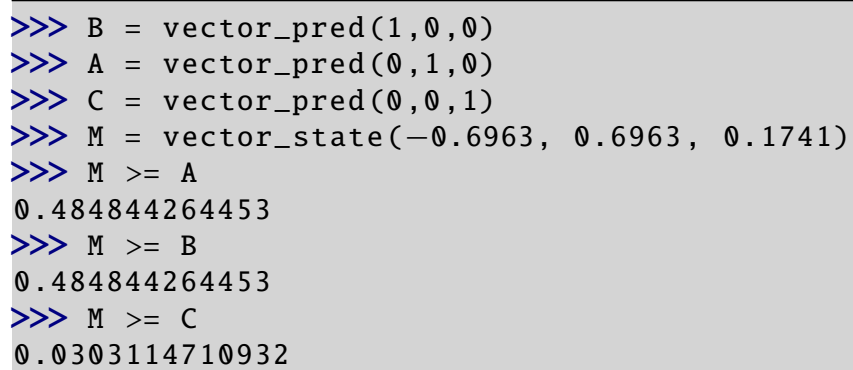

The woman's perspective is described via a different state $W$ that is defined via state transformation $\mathrm{W}=\mathrm{ch}>>\mathrm{M}$ from the man's state $\mathrm{M}$, where the channel $\mathrm{ch}$ is determined by a unitary matrix $\mathrm{U}$ :

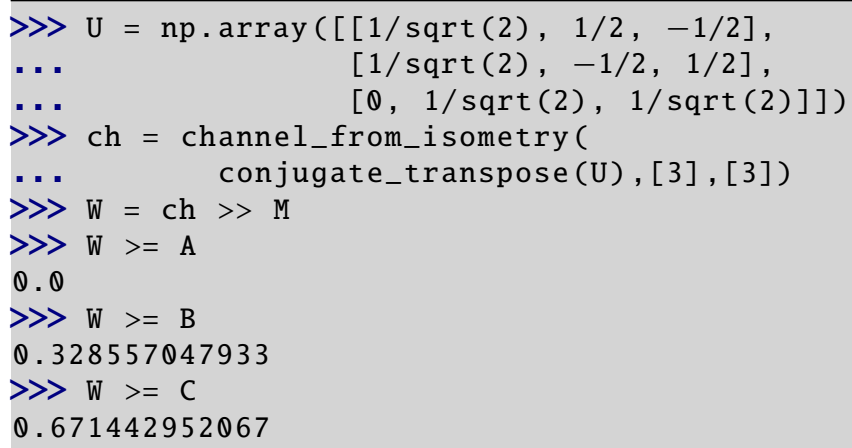

Via Eq. (10) we can also obtain these preferences of the woman in the man's state as validities of the transformed predicates:

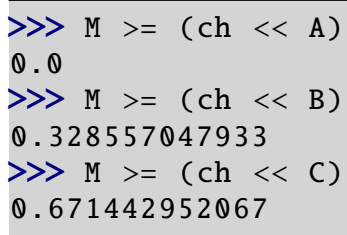

The 'order effects' arise in:

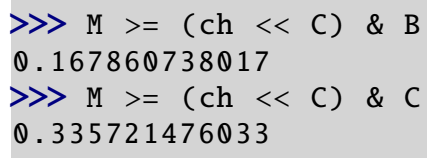

Finally, the joint choices are captured as via an update of a product state M @ W, using the EfProb notation / for conditioning.

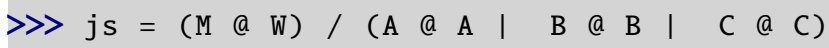

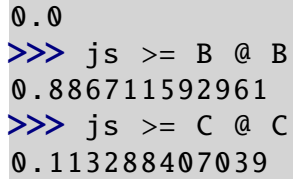

The woman's perspective originates in $\mathbb{C}^{2}$, via the channel $d$ that allows to state transformation from $\mathbb{C}^{2}$ to $\mathbb{C}^{3}$.

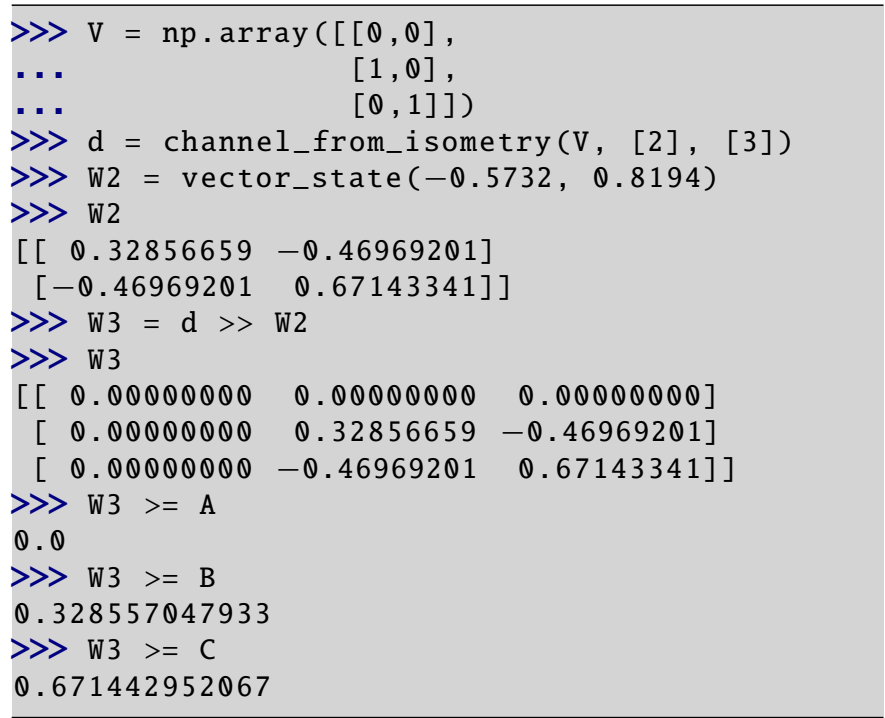

These outcomes differ slightly from the previous ones because the numbers used in the definition of the state W2 are approximations.

\section{References}

Aerts, D. (2009). Quantum structure in cognition.Journal of Mathematical Psychology, 53(5), 314-348.

Birkhoff, G., \& von Neumann, J. (1936). The logic of quantum mechanics. Annals of Mathematics, 37, 823-843.

Boole, G. (1854). An investigation of the laws of thought.

Busemeyer, J., \& Bruza, P. (2012). Quantum models of cognition and decision. Cambridge Univ. Press.

Busemeyer, J., Pothos, E., Franco, R., \& Trueblood, J. (2011). A quantum theoretical explanation for probability judgment errors. Psychological Review, 118(2), 193-218.

Cho, K., \& Jacobs, B. (2017). The EfProb library for probabilistic calculations. In F. Bonchi, \& B. König (Eds.), Conference on algebra and coalgebra in computer science (calco 2017), Vol. 72. Schloss Dagstuhl.

Cho, K., Jacobs, B., Westerbaan, A., \& Westerbaan, B. (2015). An Introduction to Effectus Theory. (see arxiv.org/abs/1512.05813).

Chovanec, F., \& Kôpka, F. (2007). D-posets. In K. Engesser, D. M. Gabbay, \& D. Lehmann (Eds.), Handbook of quantum logic and quantum structures: Quantum structures (pp. 367-428). Computer Science Press.

Dvurečenskij, A., \& Pulmannová, S. (2000). New trends in quantum structures. Dordrecht: Kluwer Acad. Publ.

Foulis, D. J., \& Bennett, M. (1994). Effect algebras and unsharp quantum logics. Foundations of Physics, 24(10), 1331-1352.

Franco, R. (2009). The conjunctive fallacy and interference effects. Journal of Mathematical Psychology, 53(5), 415-422.

Giuntini, R., \& Greuling, H. (1994). Toward a formal language for unsharp properties. Foundations of Physics, 19, 769-780.

Gudder, S. (2007). Quantum probability. In K. Engesser, D. M. Gabbay, \& D. Lehmann (Eds.), HandBook of quantum logic and quantum structures: Quantum structures (pp. 121-146). Computer Science Press.

Gudder, S., \& Greechie, R. (2002). Sequential products on effect algebras. Reports on Mathematical Physics, 49(1), 87-111.

Jacobs, B. (2015). New directions in categorical logic, for classical, probabilistic and quantum logic. Logical Methods in Computer Science, 11(3), (See https://lmcs. episciences.org/1600).

Jacobs, B., \& Cho, K. (2017). EfProb user manual. (See http://efprob.cs.ru.nl).

Kahneman, D. (2011). Thinking fast and slow. New York: Farrar, Straus and Giroux. 
Kalmbach, G. (1983). Orthomodular lattices. London: Academic Press.

Khrennikov, A. (2010). Ubiquitous quantum structure: From psychology to finances. Berlin: Springer.

Nielsen, M., \& Chuang, I. (2000). Quantum computation and quantum information. Cambridge Univ. Press.

Rieffel, E., \& Polak, W. (2011). Quantum computing. a gentle introduction. Cambridge, MA: MIT Press.
Tversky, A., \& Kahneman, D. (1983). Extension versus intuitive reasoning: The conjunction fallacy in probability judgment. Psychological Review, 90(4), 293-315.

Yearsley, J., \& Busemeyer, J. (2016). Quantum cognition and decision theories: A tutorial. Journal of Mathematical Psychology, 74, 99-116.

Zadeh, L. (1968). Probability measures of fuzzy events. Journal of Mathematical Analysis and Applications, 23(2), 421-427. 